\title{
Gap Junction Enhancer Potentiates Cytotoxicity of Cisplatin in Breast Cancer Cells
}

\author{
Ying Ding ${ }^{1}$ and Thu Annelise Nguyen ${ }^{2 *}$
}

${ }^{1}$ Department of Biochemistry, Kansas State University, Manhattan, Kansas, USA

${ }^{2}$ Department of Diagnostic Medicine/ Pathobiology, Kansas State University, Manhattan, Kansas, USA

\begin{abstract}
Cisplatin is one of the most widely used anti-cancer drugs due to its ability to damage DNA and induce apoptosis. However, increasing reports of side effects and drug resistance indicate the limitation of cisplatin in cancer therapeutics. Recent studies showed that inhibition of gap junctions diminishes the cytotoxic effect and contributes to drug resistance. Therefore, identification of molecules that counteract gap junctional inhibition without decreasing the anti-cancer effect of cisplatin could be used in combinational treatment, potentiating cisplatin efficacy and preventing resistance. This study investigates the effects of combinational treatment of cisplatin and PQ1, a gap junction enhancer, in T47D breast cancer cells. Our results showed that combinational treatment of PQ1 and cisplatin increased gap junctional intercellular communication (GJIC) as well as expressions of connexins (Cx26, Cx32 and Cx43), and subsequently decreased cell viability. Ki67, a proliferation marker, was decreased by $75 \%$ with combinational treatment. Expressions of pro-apoptotic factors (cleaved caspase-3/-8/-9 and bax) were increased by the combinational treatment with PQ1 and cisplatin; whereas, the pro-survival factor, bcl-2, was decreased by the combinational treatment. Our study demonstrates for the first time that the combinational treatment with gap junction enhancers can counteract cisplatin induced inhibition of gap junctional intercellular communication and reduction of connexin expression, thereby increasing the efficacy of cisplatin in cancer cells.
\end{abstract}

Keywords: Cisplatin; Connexin; Cytotoxicity; Gap junction; PQ1

Abbreviations: GJIC: Gap Junctional Intercellular Communication; Cx43: Connexin 43; Cx26: Connexin 26; Cx32: Connexin 32; Bax: Bcl2-Associated X Protein; Bcl-2: B-cell Lymphoma 2; DMSO: Dimethyl Sulfoxide; Cisplatin: Cis-Diamminedichloro-platinum; DAPI: 4',6-diamidino-2-phenylindole

\section{Introduction}

Cisplatin is a potent agent used in cancer chemotherapy. Since the anti-cancer properties of cisplatin were discovered in 1960s, it has been widely employed for treating various cancers, including testicular, ovarian, bladder, cervical, head and neck, esophageal, lung and breast cancer [1-4]. Numerous studies have provided information to elucidate the molecular mechanism of cisplatin cytotoxicity. It is widely accepted that the anti-tumor action of cisplatin is attributed to the formation of cisplatin-DNA adducts, inducing several signal pathways and subsequently leading to cell cycle arrest, necrosis or/and apoptosis [5]. Recently, other mechanisms without DNA-damaging effect have added to the complexity of cisplatin, including the binding of cisplatin to cellular proteins and other constituents [6]. Although cisplatin is widely used in practice due to its success in the treatment of malignancies; unfortunately, increasing drug resistance and side effects of cisplatin evoke a lot of concerns about the application [7].

Gap junctions (GJ) are intercellular channels connecting adjacent cells to allow small molecules of less than $1.2 \mathrm{kDa}$ in size to transport between cells, thereby keeping homeostasis of cells and tissues [8,9]. Many molecular processes including proliferation, differentiation, migration and apoptosis, are reported to be affected by this communication $[10,11]$. Loss of gap junctional intercellular communication (GJIC) and connexins, the gap junction proteins, is a hallmark of malignancy [12]. Connexins have been viewed as therapeutic targets in cancer treatment due to two important mechanisms: the GJIC-independent mechanism and GJIC-dependent mechanism [13]. By interacting and regulating tumor-suppressing molecules and tumor susceptible genes, connexins exhibit their tumor suppressive functions in a GJIC-independent manner [14]. A growing amount of reports suggest that over-expressing connexins can reduce cancer proliferation and attenuate tumor growth [15]. In addition to this GJIC-independent mechanism, GJ-based therapies mainly rely on the GJIC-dependent bystander effect, a mechanism by which cytotoxic molecules are transferred from target cells to neighboring cells [16]. Restoration and/or activation of GJIC have been applied in gene therapy, radiation therapy and chemotherapy [17-19]. In chemotherapy, up-regulation of GJIC and overexpression of connexins have been used to potentiate drug efficacy and reduce drug resistance [20].

Cisplatin-induced cytotoxicity has been reported to be transduced to neighboring cells through gap junctions. Jensen and Glazer found that the DNA-PK-mediated cytotoxic signal triggered by cisplatin was transmitted between cells via gap junctions [21]. The ability of activated oncogene, src, to induce cisplatin resistance by producing tyrosine phosphorylation of connexin 43 (Cx43) and decreasing GJIC, can be transmitted to adjacent cells by GJIC, even when these cells lack src activity. Moreover, this cisplatin resistant effect on neighboring cells can be counteracted by overexpression of $\mathrm{Cx} 43$ [22]. The analgesics, tramadol and flurbiprofen, used in combinational treatment with

*Corresponding author: Thu Annelise Nguyen, Kansas State University, K244 Mosier Hall, 1800 Denison Avenue, Manhattan, KS 66506, USA, Tel: 785-5324429; Fax: 785-532-4039; E-mail: tnguyen@vet.k-state.edu

Received September 20, 2012; Accepted October 23, 2012; Published October 25,2012

Citation: Ding Y, Nguyen TA (2012) Gap Junction Enhancer Potentiates Cytotoxicity of Cisplatin in Breast Cancer Cells. J Cancer Sci Ther 4: 371-378. doi:10.4172/1948-5956.1000170

Copyright: @ 2012 Ding Y, et al. This is an open-access article distributed under the terms of the Creative Commons Attribution License, which permits unrestricted use, distribution, and reproduction in any medium, provided the original author and source are credited. 
cisplatin, were shown to depress the cytotoxicity of cisplatin via the inhibition effects on gap junctions [23]. Furthermore, cisplatin was reported to inhibit GJIC by directly inhibiting the channel activity and decreasing expression of connexins [24]. This evidence indicates that inhibition of GJIC and reduction of connexins would decrease cytotoxicity of cisplatin and result in cisplatin resistance. Therefore, development of novel agents or methods to enhance or restore GJIC in combinational treatment with cisplatin is a new strategy to potentiate cisplatin effect and decrease drug resistance.

PQ1, a derivative of quinoline, was reported as a gap junction enhancer [25]. Gakhar et al. [25] reported that $200 \mathrm{nM}$ of PQ1 showed a significant increase in the GJIC in T47D breast cancer cells. Combinational treatment of PQ1 and tamoxifen indicated that PQ1 potentiated the effect of tamoxifen in T47D cells, indicating the synergistic effect of PQ1 in combinational treatment in breast cancers [26].

In this report, the effects of PQ1 on the cytotoxicity of cisplatin in breast cancer cells were examined. Our results showed that PQ1 counteracted the inhibition of GJIC and reduction of connexins caused by cisplatin, subsequently enhancing the cytotoxic effect of cisplatin.

\section{Materials and Methods}

\section{Reagents and antibodies}

PQ1, a quinoline derivative, was obtained as described by Shi et al. [27] and graciously provided by Dr. Duy Hua (Kansas State University). Cis-Diamminedichloro-platinum, trypan blue, Lucifer yellow dye and Rhodamine-dextran dye were all purchased from Sigma (St Louis, MO, USA). Anti-Cx43, Alexa-568-conjugated anti-rabbit IgG, and Alexa-594-conjugated anti-mouse IgG antibodies were obtained from Invitrogen (Camarillo, CA, USA). Anti-Cx32, anti-Cx26, anti-Ki67, anti-cleaved caspase-3, anti-caspase- 8 p18, anti-caspase- 9 p35, antiBax, and anti-Bcl2 antibodies were all purchased from Santa Cruz Biotechnologies (Santa Cruz, CA, USA). The HRP-linked anti-rabbit/ mouse antibodies were purchased from Cell Signaling Technology (Danvers, MA, USA).

\section{Cell line and cell culture}

The T47D human breast cancer cell line was purchased from American Type Cell Culture (ATCC) (Manassas, MA, USA). The cells were grown in RPMI-1640 (Sigma-Aldrich, St Louis, MO, USA) supplemented with $2 \mathrm{~g} / \mathrm{L}$ sodium bicarbonate, $1 \mathrm{mM}$ sodium pyruvate, $10 \mathrm{mM}$ hepes, $4.5 \mathrm{~g} / \mathrm{L}$ glucose, 0.2 units/ml bovine insulin, and $10 \%$ fetal bovine serum (Atlanta biological, Lawrenceville, GA, USA). Cells were maintained in $\mathrm{T}-75 \mathrm{~cm}^{2}$ flasks at $37^{\circ} \mathrm{C}$ with $5 \% \mathrm{CO}_{2}$ and cultured in 6-well plates or T-25 $\mathrm{cm}^{2}$ flasks for experimental analysis.

\section{Cell morphology}

T47D cells were cultured in six-well plates until $80 \%$ confluent state and treated according to the following conditions: untreated, PQ1 $(100,200$, and $500 \mathrm{nM})$ alone for 28 hours, cisplatin $(40 \mu \mathrm{M})$ alone for 24 hours, and PQ1 (100, 200, and $500 \mathrm{nM})$ for 4 hours followed by addition of cisplatin for 24 hours. Cell morphology was captured using Nikon 80i light microscope.

\section{Cell viability assay}

Cell viability was measured using trypan blue excision method. T47D cells were cultured into 6-well plates until $80 \%$ confluent state (high density) or $40 \%$ confluent state (low density), corresponding to the conditions in which junctional channel formation was permitted or not, respectively. Cells were treated with PQ1 and cisplatin as discussed for the cell morphology protocol. Cells floated in the media were collected and cells attached to the wells were trypsinized. Two parts of cells were combined together, centrifuged and resuspended. A cell suspension was mixed with trypan blue dye and viable cells were examined by the Cellometer Auto 2000 (Nexcelom Bioscience, Lawrence, MA, USA).

\section{Scrape load/ dye transfer assay}

T47D cells were seeded on coverslips in 6-well plates and cultured until the confluency reached $80 \%-100 \%$. Cells were treated with PQ1 $(100,200$, and $500 \mathrm{nM})$ and cisplatin $(40 \mu \mathrm{M})$ alone for 4 hours or sequential combinational treatment, treating PQ1 for 4 hours followed by cisplatin for 4 hours. After treatments, cells were rinsed three times with PBS. Then, $2.5 \mu \mathrm{l}$ mixtures of $1 \%(\mathrm{w} / \mathrm{v})$ Lucifer yellow and $1 \%$ $(\mathrm{w} / \mathrm{v})$ Rhodamine-dextran was added in the center of the coverslips and a scrape was made on the coverslips. The dye solution was left on the cover slips for 3 minutes, after which coverslips were washed by PBS for three times. The cells were incubated in RPMI medium at $37^{\circ} \mathrm{C}$ for 20 minutes, washed by PBS, and then fixed with $2.5 \%$ paraformaldehyde for 10 minutes. Cells were mounted and image of dye transfer was captured using Nikon TE2000U fluorescence microscope. The distance of dye transfer from cutting site to the farthest visual uptake of dye was measured using ZEN 2010 software.

\section{Western blot analysis}

T47D cells were cultured in T-25 $\mathrm{cm}^{2}$ flasks until $80 \%$ confluent state. Cells were treated with PQ1 alone for 28 hours, cisplatin alone for 24 hours, or PQ1 for 4 hours followed by addition of cisplatin for 24 hours. Cells without any treatments or DMSO as vehicle were used as controls. After treatments, cells were washed with PBS for three times and harvested in lysis buffer (Cell Signaling Technology, Danver, MA, USA). Cell lysates were sonicated using Vibra-Cell sonicator (Sonics \& Materials Inc, Danbury, CT, USA) and then centrifuged at 13,000 $\mathrm{rpm}$ for 30 minutes at $4^{\circ} \mathrm{C}$. After centrifugation, supernatants were collected as whole cell extracts. Thirty ug of samples were separated by 4-20\% gradient SDS-PAGE for 35 minutes at $200 \mathrm{~V}$, and transferred to nitrocellulose membranes (Midwest Scientific, Saint Louis, MO, USA). Membranes were blocked with 5\% milk for 30 minutes and immunoblotted against protein of interest. Immunoreactions using chemiluminescence were visualized by FluorChem E Imaging Instrument (ProteinSimple, Santa Clara, CA, USA). Intensities of the bands were digitized using Un-Scan-It software.

\section{Immunofluorescence and confocal microscopy}

T47D cells cultured on coverslips in 6-well plates were treated with PQ1 and cisplatin alone or in combination as described in Western blot analysis protocol. After treatment, cells were rinsed with PBS and fixed with $2 \%$ paraformaldehyde for 20 minutes at room temperature. Fixed cells were washed 3 times with PBS and then permeabilized with $0.1 \%$ Triton X-100 for 8 minutes. Cells were washed 3 times with PBS again, and blocked with $2.5 \%$ BSA in PBS for 1 hour at room temperature. After blocking, cells were incubated with primary antibodies overnight at $4^{\circ} \mathrm{C}$. Samples were incubated with Alexa-conjugated secondary antibodies for 1 hour at room temperature. DAPI was used to stain nuclei. The slides were mounted by prolong-antifade reagent (Invitrogen, Camarillo, CA, USA) and sealed. Image was captured using a confocal microscope (Carl Zeiss LSM 700 META, Narashige, MN, USA). 
Citation: Ding Y, Nguyen TA (2012) Gap Junction Enhancer Potentiates Cytotoxicity of Cisplatin in Breast Cancer Cells. J Cancer Sci Ther 4: 371378. doi:10.4172/1948-5956.1000170

\section{Statistical analysis}

Data were statistically analyzed by using student's t-test. Data presented were expressed as mean \pm S.D. of at least three independent experiments. Significance was considered at $\mathrm{p}<0.05$.

\section{Results}

Combinational treatment of PQ1 and cisplatin has a synergistic effect on cell morphology and proliferation in T47D cells

Cisplatin and PQ1 separately have been reported to inhibit cell proliferation and induce cell death in breast cancer cells via different mode of action [25,28]. To address whether cisplatin and PQ1 can work synergistically to attenuate cell proliferation, cell morphology and proliferation were first examined with the combinational treatment of PQ1 and cisplatin. T47D breast cancer cells were treated with PQ1 and cisplatin alone and in combination as PQ1 for 4 hours followed by addition of cisplatin for 24 hours. Cells without any treatment were used as controls. Morphological evaluation showed that both PQ1 and cisplatin changed cell morphology and decreased the number of adherent cells (Figure 1). Combinational treatment of PQ1 and cisplatin induced more significant morphological changes compared to PQ1 and cisplatin alone. Changes including irregular shape, shrinkage, rounding, and detachment were found in PQ1-pretreated cells in a dose-dependent manner (Figure 1). Combinational treatment of T47D cells with $40 \mu \mathrm{M}$ cisplatin and 200 or $500 \mathrm{nM}$ PQ1 caused a significant change in the morphology with less than $50 \%$ of cells attached to the bottom of flasks (Figure 1). The proliferation of cells was examined by the staining of Ki67, a nuclear protein as a proliferation marker. 24 hours treatment of $40 \mu \mathrm{M}$ cisplatin did not significantly decrease expression of Ki67, indicating that 24 hours treatment of cisplatin is not sufficient for the inhibition of cell proliferation (Figures 2A \& 2B). However, Ki67 staining was decreased in the presence of PQ1

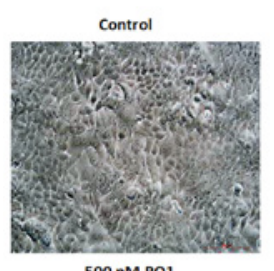

$500 \mathrm{nM}$ PQ1

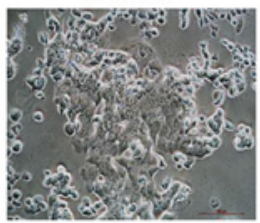

$100 \mathrm{nM} P \mathrm{PQ}_{1}+40 \mu \mathrm{M}$ cisplati

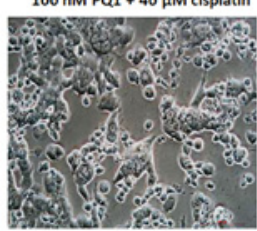

$100 \mathrm{nM}$ PQ1

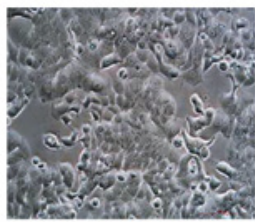

$40 \mu \mathrm{M}$ cisplatin

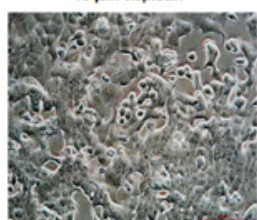

$200 \mathrm{nM}$ PQ1 $+40 \mu \mathrm{M}$ cisplatin

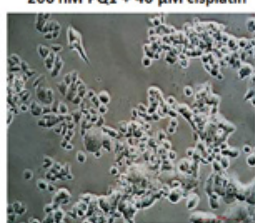

$200 \mathrm{nM}$ PQ1

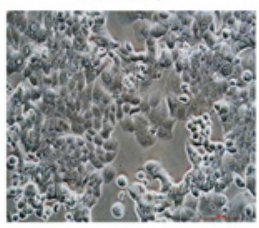

DMSO + $40 \mu \mathrm{M}$ cisplatin

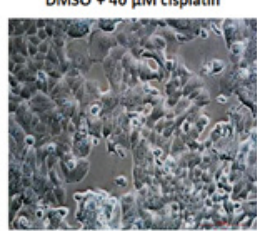

$500 \mathrm{nM}$ PQ1 $+40 \mu \mathrm{M}$ cisplatin

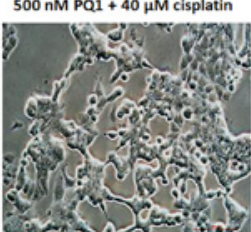
gistic effect on cell morphology T47D cells were treated with PQ1 and cisplatin alone and in combination as indicated. Cells without treatment were used as controls. DMSO, a PQ1 vehicle, was used as solvent control. Cell morphology was captured using the light microscope under 20X magnification. The scale bar is $100 \mu \mathrm{m}$ in size.

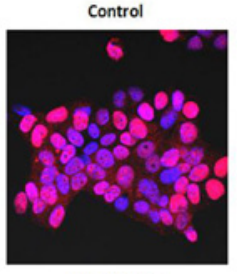

$500 \mathrm{nM}$ PQ1

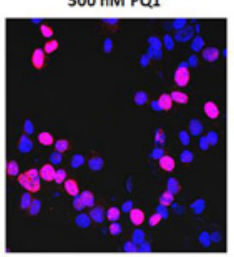

$100 \mathrm{nM}$ PQ1 $+40 \mu \mathrm{M}$ cisplatin

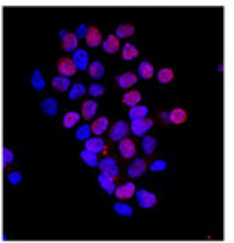

$100 \mathrm{nM} P Q 1$

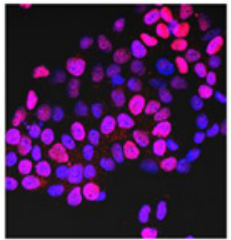

$40 \mu \mathrm{M}$ cisplatin

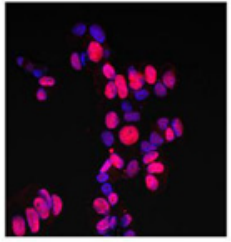

$200 \mathrm{nM}$ PQ1 $+40 \mu \mathrm{M}$ cisplatin

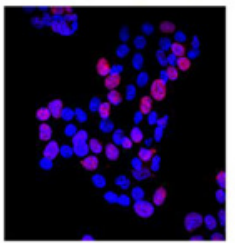

$200 \mathrm{nM}$ PQ1

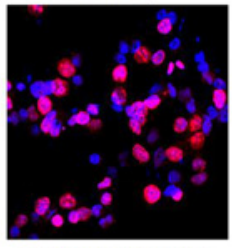

DMSO + $40 \mu \mathrm{M}$ cisplatin

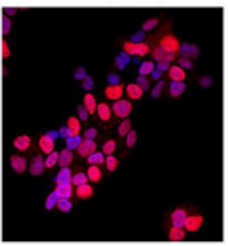

$500 \mathrm{nM}$ PQ1 $+40 \mu \mathrm{M}$ cisplatin

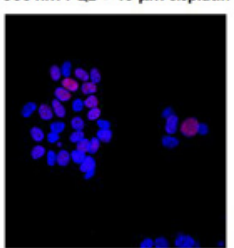

B

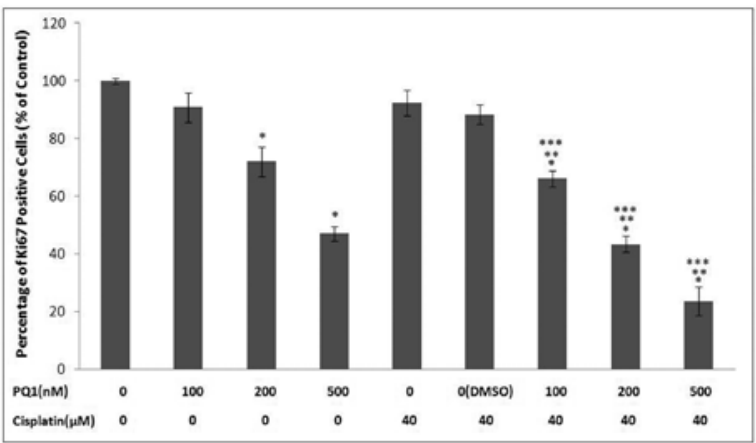

Figure 2: Combinational treatment of $P Q 1$ and cisplatin has a synergistic effect on cell proliferation. T47D cells were treated with PQ1 and cisplatin alone and in combination. (A) Ki67 staining was used to examine cell proliferation under confocal microscope. Red indicates Ki67 and blue indicates nuclei stained by DAPI. (B) Percentages of Ki67 in treated cells were calculated and the results of each treatment were normalized to its controls. Data were obtained in three independent experiments and are represented as the mean $\pm \mathrm{S}$.E. * $\mathrm{P}$-value is $<0.05$ compared to control. ${ }^{*} \mathrm{P}$-value is $<0.05$ compared to cisplatin treatment. ${ }^{* *} \mathrm{P}$-value is $<0.05$ compared to $\mathrm{PQ} 1$ treatment.

in a dose-dependent manner. The combinational treatment of 500 nM PQ1 and $40 \mu \mathrm{M}$ cisplatin has a significant decrease of $23.6 \%$ of Ki67 stained cells compared to $92.5 \%$ with cisplatin alone and $47.1 \%$ with PQ1 alone (Figure 2B), indicating a synergistic effect of the combinational treatment on anti-proliferation. DMSO, a PQ1 vehicle, was used as solvent control, showing no changes on cell morphology and proliferation induced by the solvent (Figures 1, 2A \&2B). These results suggest that combinational treatment of $\mathrm{PQ} 1$ and cisplatin has a synergistic effect on the changes of cell morphology and the inhibition of cell proliferation.

PQ1 increases cytotoxicity of cisplatin and the enhancement depends on cell density

The cytotoxicity of cisplatin in part is due to the bystander effect 
via GJIC at high cell density [24]. Cisplatin-induced cytotoxicity is transduced to neighbor cells through gap junction and accordingly induces more cell death under high cell density condition [24]. Since PQ1 has been reported as a gap junction enhancer [25], the effect of PQ1 on GJIC raised the possibility that the cytotoxicity of cisplatin could be potentiated by PQ1 via GJIC mediation. Here, cytotoxicity of combinational treatment was examined under low and high cell density conditions. At low density, cells were well dispersed as single cells without touching the neighboring cells, in which a condition with few gap junctions could be formed. However, at high density, cells were confluent enough to contact with adjacent cells, allowing the formation of gap junctions. Consistent with previous reports [24], the density-dependent toxicity of cisplatin was also observed in this study. 24 hours treatment of cisplatin decreased cell viability to $85 \%$ at low density and $75 \%$ at high density, indicating that the cytotoxicity of cisplatin is GJIC-dependent (Figures 3A \& 3B). PQ1 enhanced the cytotoxicity of cisplatin at low and high density in a concentrationdependent manner, but the increase of toxic effects at high density is more significant (Figures 3A, 3B \& 3C). Compared with cisplatin treatment alone, a $33 \%$ decrease $(75 \%$ to $42 \%$ ) of cell viability was observed in the combinational treatment of cisplatin and $500 \mathrm{nM}$ PQ1 under high density condition; whereas, same combinational treatment only caused a $24 \%$ decrease ( $85 \%$ to $61 \%$ ) under low density condition (Figures 3A, 3B \& 3C). Relative to cisplatin treatment alone, quantification of differences in survival, caused by the combinational treatment at different PQ1 concentrations between low and high densities, were showed in figure 3C. PQ1 had a constant effect on cell toxicity regardless on cell density (Figures $3 \mathrm{~A} \& 3 \mathrm{~B}$ ). Therefore, the fact that PQ1 had a greater effect on the cytotoxicity of cisplatin at high density suggested an enhanced toxic effect of cisplatin via up-regulated GJIC mediated by PQ1.

\section{PQ1 counteracts cisplatin inhibition of GJIC and reduction of connexins}

Effects of PQ1 on GJIC mediation in the combinational treatment was further investigated by examining the activity of the gap junction channels and the expression of connexins. From previous findings, 24 hours treatment with PQ1 and cisplatin can cause cell death, which subsequently reduces the cell density and gap junctions (Figures 1, 3A, $\& 3 \mathrm{~B})$. Therefore, 4 hours incubation was used to avoid the substantial cell death in the SL/DT experiment. T47D cells were treated with PQ1 and cisplatin alone or in combination, and then scrap load/dye transfer (SL/DT) was performed. As a gap junction enhancer, PQ1 significantly increased GJIC. $500 \mathrm{nM}$ PQ1 increased the distance of dye transfer by 3.7 times compared to control (Figure $4 \mathrm{~B}$ ). In contrast, cisplatin treatment alone decreased $60 \%$ of the dye transfer from cell to cell compared to control, indicating that cisplatin can cause an inhibition of GJIC (Figure 4B). When cells were incubated with PQ1 prior to cisplatin treatment, a decrease of dye transfer induced by cisplatin was rescued (Figures 4A \& 4B). Combinational treatment of $500 \mathrm{nM}$ PQ1 and cisplatin caused a 7.4-fold increase in the distance of dye transfer compared with cisplatin treatment alone, as well as a 2.6-fold increase of dye transfer compared to control (Figure 4B). This implies that not only does $500 \mathrm{nM}$ PQ1 restore cisplatin-inhibited GJIC, but also increases the overall GJIC activity in T47D cells. The dye transfer increased with an increase in PQ1, suggesting that PQ1-mediated GJIC is concentration dependent (Figures 4A \& 4B).

Gap junctional proteins such as connexin 26 (Cx26), connexin
A

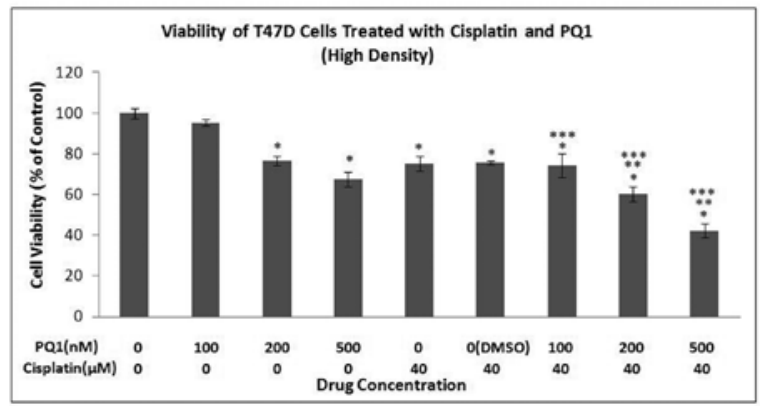

B

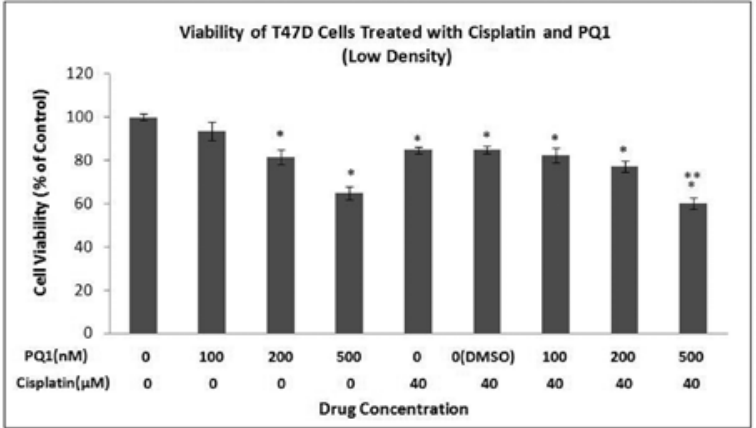

C

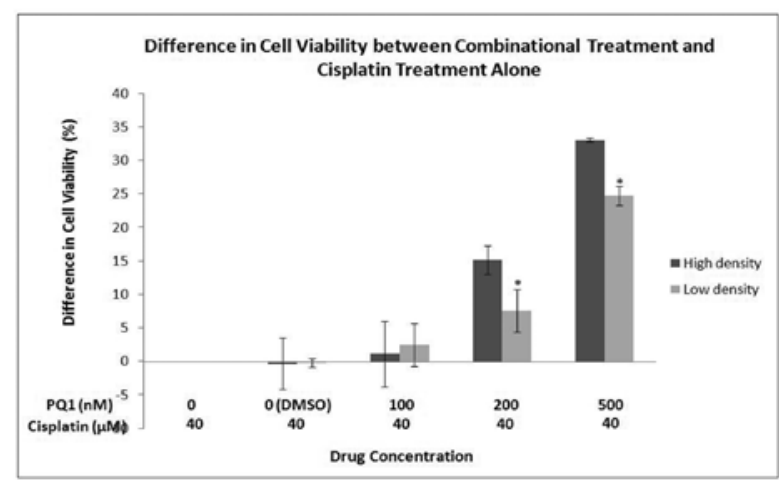

Figure 3: PQ1 increases cytotoxicity of cisplatin and the enhancement depends on cell density. T47D cells were treated with PQ1 and cisplatin alone or in combination as indicated. Cell viability was measured under high density (A) and low density (B). Graphical presentation of three independent experiments is presented with statistical significance. * P-value is $<0.05 \mathrm{com}$ pared to control. " $\mathrm{P}$-value is $<0.05$ compared to cisplatin treatment. ${ }^{* * *} \mathrm{P}$ value is $<0.05$ compared to $P Q 1$ treatment. (C) Quantification of differences in survival, caused by PQ1 at different concentrations in the combinational treatment between low density and high density. The viabilities of cells treated with cisplatin alone at high and low density were used as controls. Data were obtained in three independent experiments and are represented as the mean \pm S.E. * P-value is $<0.05$ compared to treatment at high density.

$32(\mathrm{Cx} 32)$, and connexin $43(\mathrm{Cx} 43)$ are reported to express in human breast cancer cells [29]. Here, levels of these connexins were measured by Western blotting. The results showed that $500 \mathrm{nM}$ PQ1 alone increased the levels of $\mathrm{Cx} 26, \mathrm{Cx} 32$ and $\mathrm{Cx} 43$ by $44 \%, 55 \%$ and $18 \%$, whereas $40 \mu \mathrm{M}$ cisplatin alone showed a decrease in $\mathrm{Cx} 26, \mathrm{Cx} 32$ and $\mathrm{Cx} 43$ by $47 \%, 54 \%$, and $42 \%$, respectively. These indicate that the effect of PQ1 and cisplatin treatment is on the level of connexin expression (Figure 5B). When cells were treated with both PQ1 and cisplatin, the downregulation of connexin by cisplatin was reversed by PQ1 (Figures 5A \& 5B). Relative to cisplatin alone, combinational treatment of $500 \mathrm{nM} P Q 1$ and cisplatin significantly increased Cx26, Cx32, and Cx43 by $151 \%, 189 \%$, and $74 \%$, respectively (Figure 5B). These results provide evidence that PQ1 can rescue cisplatin-induced 


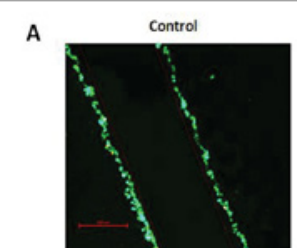

$500 \mathrm{nM}$ PQ1

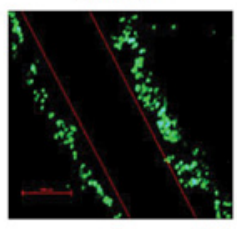

$100 \mathrm{nM} P Q 1+40 \mu$ M cisplatin

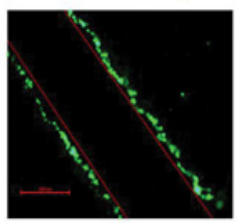

B

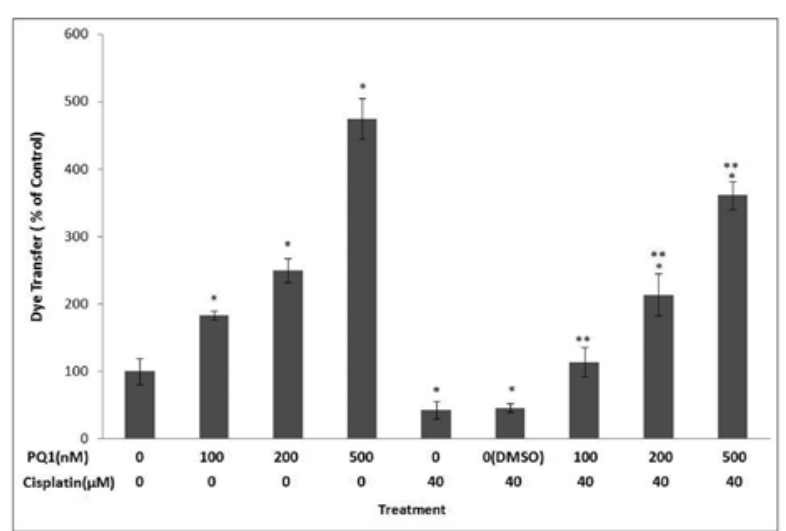

Figure 4: PQ1 counteracts cisplatin inhibition of GJIC. T47D cells were treated with PQ1 and cisplatin alone and in combination as indicated. Cells without treatment were used as controls. (A) Scrape load/dye transfer assay was performed. Green indicates lucifer yellow and red indicates rhodaminedextran. Gap junction activity is examined by measuring the distance of Lucifer yellow dye transfer. Image of dye transfer was captured using a fluorescence microscope under $4 \mathrm{X}$ magnification. The scale bar is $100 \mu \mathrm{m}$ in size (B) The distance of dye transfer from cutting edge to the farthest cells with the dye uptake was measured using Zen 2010 software. The distance of dye transfer for treated cells was normalized to the distance of dye transfer of its control group and the graphical presentation of three independent experiments was showed with statistical significance. * P-value is $<0.05$ compared to control. $\mathrm{P}$-value is $<0.05$ compared to cisplatin treatment.

connexin downregulation and thus subsequently increase the overall GJIC activity in T47D cells.

\section{Combinational treatment of PQ1 and cisplatin enhances apoptosis}

Cisplatin is involved in multiple mechanisms after the formation of cisplatin-DNA adducts to induce cell death [6]. One mechanism is the induction of apoptosis, a programmed cell death [30]. The intrinsic and extrinsic pathways of apoptosis are reported to be activated in response to cisplatin in different cell lines. In human osteosarcoma, cisplatin activates caspase- 8 , the initiator of extrinsic pathway, and subsequently activates caspase- 3 to cause cell death [31]. However, in many other cell lines (like human SCC-25 squamous carcinoma and cisplatinsensitive human testicular cancer cell lines), cisplatin was found to activate the intrinsic pathway of apoptosis by releasing cytochrome $c$ and sequentially activating caspase-9 (the intrinsic initiator) and caspase-3 $[32,33]$. In this study, both intrinsic and extrinsic pathways of apoptosis were examined to evaluate the effect of combinational treatment on apoptosis.

T47D cells were treated with PQ1 and cisplatin individually or in combination as described in Materials and Methods. Activation of caspase- 8 and -9 were determined by Western blotting using antibodies specific to cleaved caspase- 9 subunit p35 (caspase-9 p35) and cleaved caspase- 8 subunit p18 (caspase-8 p18). Our results showed that 24 hours treatment of $40 \mu \mathrm{M}$ cisplatin did not significantly change the level of caspase- 9 p35, but slightly increased the level of caspase- 8 p18 by $13 \%$, indicating that cisplatin induced apoptosis through extrinsic pathway within 24 hours (Figures 6A \& 6B). However, 500 nM PQ1 increased the levels of both cleaved caspase- 8 and -9 , indicating that the activation of both pathways can be induced by PQ1 (Figures 6A \& 6B). Interestingly, the combinational treatment of $500 \mathrm{nM}$ PQ1 and $40 \mu \mathrm{M}$ cisplatin had a synergistic effect on both caspase- 8 and -9 compared to the treatment of PQ1 or cisplatin alone. The relative percentage of caspase-8 p18 expression for the combinational treatment of $500 \mathrm{nM}$ PQ1 and $40 \mu \mathrm{M}$ cisplatin is $185 \%$ compared to $113 \%$ for cisplatin and $138 \%$ for PQ1 (Figure 6B). Similarly, the combinational treatment also significantly increased the relative percentage of caspase- 9 p35 expression to $149 \%$ compared to $94 \%$ for cisplatin and $124 \%$ for PQ1 (Figure 6B).

To further investigate the intrinsic pathway, expression of two important effectors: $\mathrm{Bcl}-2$, an anti-apoptotic protein, and Bax, a proapoptotic protein, were examined. The ratio of Bcl-2 to Bax is crucial to the release of cytochrome $\mathrm{c}$ and subsequently determines if the cell will enter the execution phase [34]. Cisplatin is reported to increase the levels of Bax and keep the expression of Bcl-2 unchanged in cisplatin-

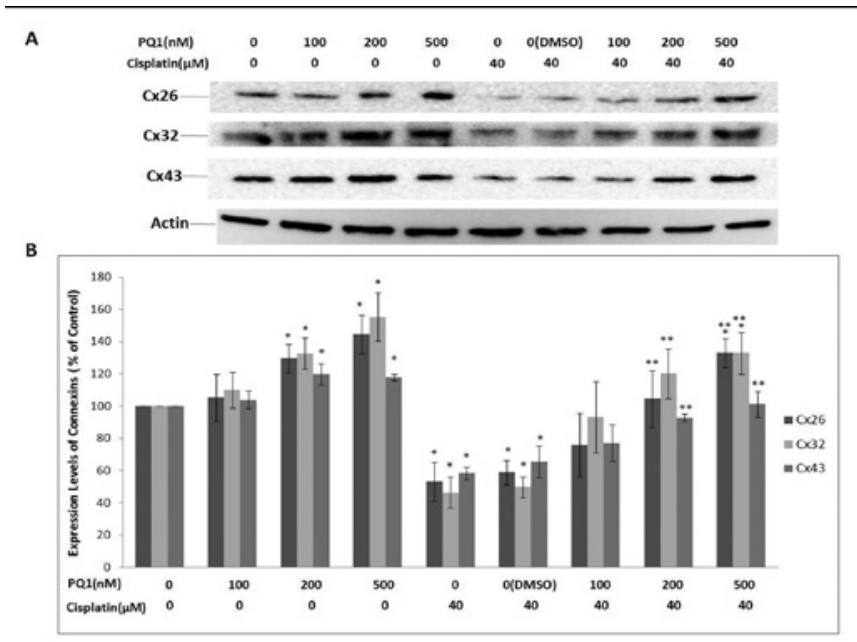

Figure 5: PQ1 counteracts cisplatin reduction of connexins. T47D cells were treated with $P Q 1$ and cisplatin alone and in combination as described. (A) Expression of connexin 26 (Cx26), connexin 32 (Cx32), and connexin 43 (Cx43) were examined by Western blot analysis. Actin was used as loading control. (B) Graphical presentation of three independent experiments shows levels of connexins normalized to control. Pixel intensities of protein bands were normalized to pixel intensities of actin, and the results of treated cells are normalized to the results of the controls. * P-value is $<0.05$ compared to control. " $\mathrm{P}$-value is $<0.05$ compared to cisplatin treatment. 
Citation: Ding Y, Nguyen TA (2012) Gap Junction Enhancer Potentiates Cytotoxicity of Cisplatin in Breast Cancer Cells. J Cancer Sci Ther 4: 371378. doi:10.4172/1948-5956.1000170

sensitive ovarian cells $[35,36]$. However, in some cisplatin-resistant ovarian cells, cisplatin-treated cells overexpress $\mathrm{Bcl}-2$ and the high level of Bcl-2 protects cells from apoptosis by suppressing Bax [37]. Our results showed that 24 hours treatment of $40 \mu \mathrm{M}$ cisplatin did not significantly change the level of Bax and Bcl-2, compared to control (Figure 6B). 200 and 500 nM PQ1 increased the level of Bax, but had no significant effect on Bcl-2. Interestingly, the combinational treatment with PQ1 and cisplatin synergistically increased Bax and decreased Bcl-2. Combinational treatment with $500 \mathrm{nM}$ PQ1 and $40 \mu \mathrm{M}$ cisplatin increased the level of Bax to $189.8 \%$ compared to $101.9 \%$ for cisplatin alone and $127.7 \%$ for PQ1 alone. However, the combinational treatment decreased the level of Bcl-2 to $23.3 \%$ compared to $119.8 \%$ for cisplatin alone and $101.6 \%$ for PQ1 alone (Figure 6B). The results of Bax and Bcl-2 are consistent with the expression of caspase- 9 p35, indicating that an activation of intrinsic pathway can be induced by the combinational treatment of PQ1 and cisplatin.

Caspase-3 is an executioner at the convergence of multiple apoptotic signaling pathways, and activation of caspase- 3 is considered to be the last step of apoptosis. The apoptotic effect of combinational treatment was further investigated by examining the expression of cleaved caspase- 3 using confocal microscopy. The staining of cleaved caspase- 3 was faint with $40 \mu \mathrm{M}$ cisplatin for 24 hours treatment (Figure 6C). After combinational treatment of 200 or $500 \mathrm{nM}$ PQ1 and 40 $\mu \mathrm{M}$ cisplatin, a significant increase of cleaved caspase- 3 staining was detected (Figure 6C). All the results of apoptotic factors suggest that the combinational treatment with PQ1 and cisplatin greatly enhanced apoptosis by activating both intrinsic and extrinsic pathways.

\section{Discussion}

Drug resistance and detrimental side effects are two major problems in platinum-based chemotherapy in cancer treatment [38] Many mechanisms have been reported to contribute to cisplatin resistance, including DNA repair, signaling pathway regulation, and tumor microenvironment modulation [7]. In addition to these widelyaccepted mechanisms which have been studied for many years, recent studies found that cisplatin-induced resistance is also associated with deficiency in cell-cell communication, the GJIC [7]. Deficiency of
A
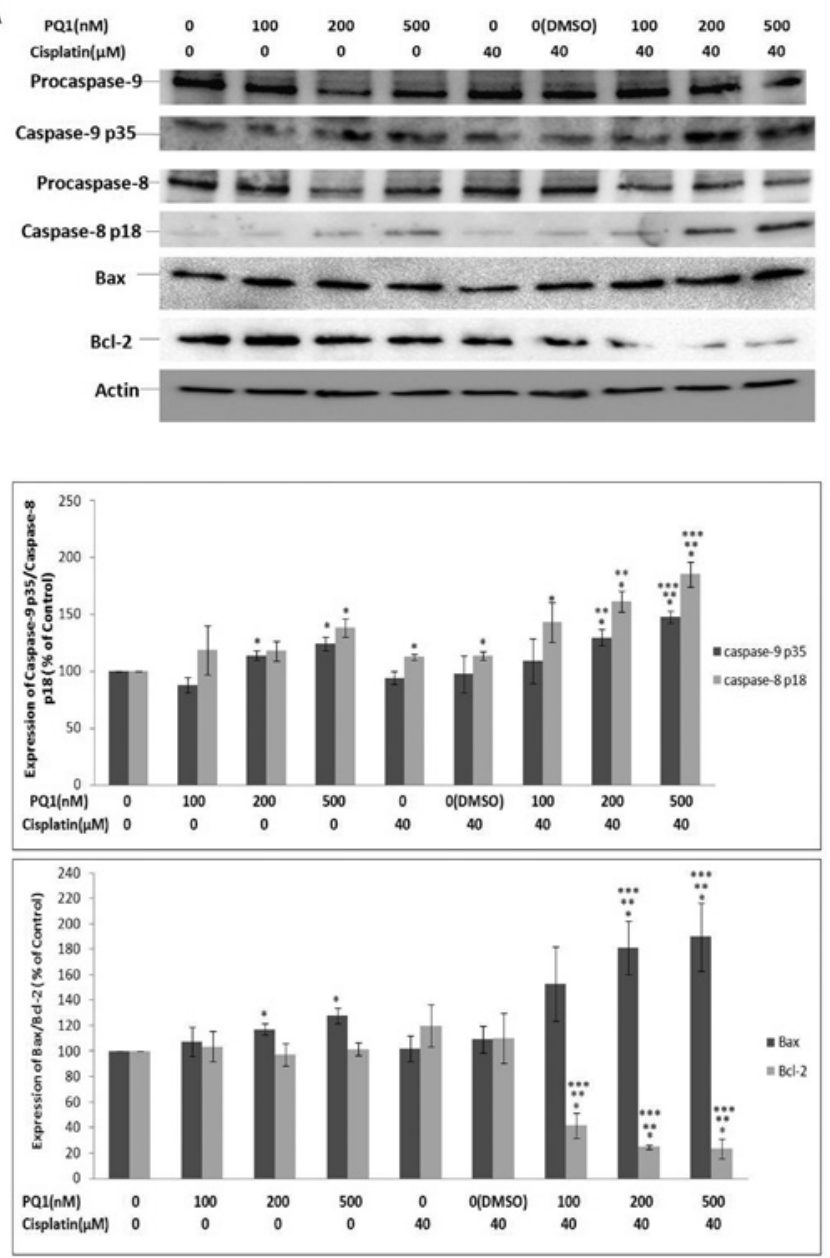

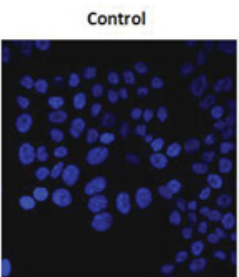

$500 \mathrm{nM} P Q 1$

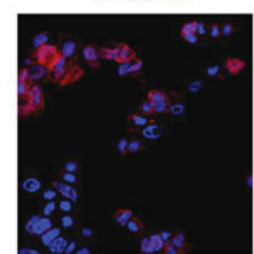

$100 \mathrm{nM}$ PQ1 $+40 \mu \mathrm{M}$ cisplatin

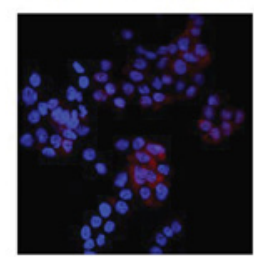

$100 \mathrm{nM}$ PQ1

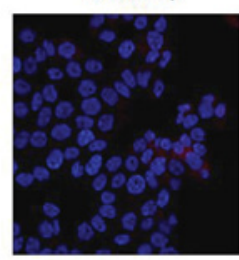

$40 \mu \mathrm{M}$ cisplatin

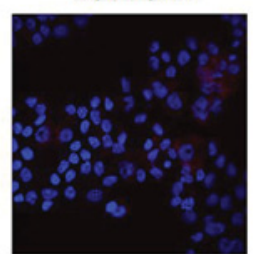

$200 \mathrm{nM}$ PQ1 $+40 \mu \mathrm{M}$ cisplatin

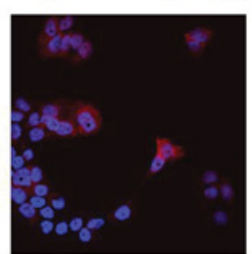

$200 \mathrm{nM} P Q 1$

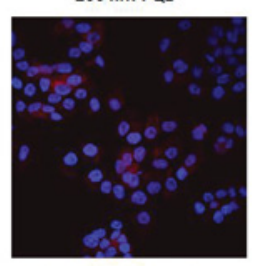

DMSO $+40 \mu \mathrm{M}$ cisplatin

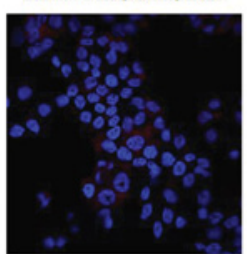

$500 \mathrm{nM} P Q 1+40 \mu \mathrm{M}$ cisplatin

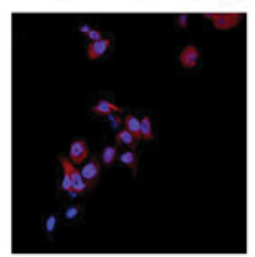

Figure 6: Combinational treatment of PQ1 and cisplatin enhances apoptosis. T47D cells were treated with PQ1 and cisplatin alone and in combination as indicated. Cells without treatment were used as controls. (A) Expression of procaspase-9, caspase-9 p35, procaspase-8, caspase-8 p18, Bax and Bcl-2 were examined by Western blot analysis. Actin was used as loading control. (B) Graphical presentation of three independent experiments shows levels of apoptotic factors normalized to control. Pixel intensities of protein bands were normalized to pixel intensities of actin, and the results of treated cells are normalized to the results of the controls. * $\mathrm{P}$-value is $<0.05$ compared to control. " $\mathrm{P}$-value is $<0.05$ compared to cisplatin treatment. ${ }^{* * *} \mathrm{P}$-value is $<0.05$ compared to $\mathrm{PQ} 1$ treatment. (C) Immunofluorescence was performed to examine expression levels of cleaved caspase-3. Red is cleaved caspase- 3 and blue indicates the nuclei. 
GJIC in cancer cells and inhibition of GJIC by cisplatin depress the cytotoxicity of cisplatin by preventing the cytotoxic molecules or signals from spreading throughout a tumor mass. Therefore, strategies to regulate gap junctions are needed to circumvent or decrease cisplatin resistance.

PQ1, a derivative of quinoline, has been reported to enhance GJIC, inhibit cell and tumor growth, and increase potential of combinational treatment with tamoxifen in T47D breast cancer cells [25,26]. Studies about the effects of PQ1 on normal tissues further showed that PQ1 administration can be achieved with low toxicity to normal organs [39]. All these results indicate that PQ1 is a promising agent in GJbased cancer therapy. The present study investigated the influence of PQ1 on the cytotoxicity of cisplatin in T47D breast cancer cells. The results showed that combinational treatment of PQ1 and cisplatin counteracted the cisplatin-induced inhibition of GJIC and decrease of connexins, and subsequently enhanced cytotoxic effects.

Formation and degradation of gap junctions are dynamic processes with a half-life of connexin not exceeding 5 hours [40]. Therefore, short-term pretreatment is used in current experiments, examining the effect of communication activity before the degradation of connexins. Current study demonstrated that 4 hours treatment is sufficient for PQ1-increasing GJIC in T47D cells. To optimize the activation of GJIC by PQ1 as well as minimize the inhibition of GJIC by cisplatin, PQ1 was dosed 4 hours prior to cisplatin in the combinational treatment.

The GJIC-mediated bystander effect has been demonstrated to play an important role in transferring toxic effects. For example, the application of bystander effect in gene therapy showed that after enhancing connexin $43(\mathrm{Cx} 43)$ and GJIC by 8-bromo-cyclic-AMP treatment the toxic effect was strengthened by herpes simplex virus thymidine kinase/gancyclovir (HSV-TK/GCV) system [17]. Consistent with previous reports [24], our studies showed that the toxicity of cisplatin was greater at high density when there is opportunity for gap junctional contacts between the cells (Figures 3A \& 3B), indicating that the cytotoxicity of cisplatin is mediated by GJIC. Therefore, inhibition of gap junctional activity and reduction of connexin expressions by cisplatin (Figures $4 \& 5$ ) may be in part the cause of low cisplatin cytotoxicity in T47D cells. The present data showed that 4 hours pretreatment of PQ1 counteracted inhibition of GJIC induced by cisplatin (Figure 4). The direct effect of PQ1 on GJIC suggested a GJICdependent mechanism for the effect of PQ1 on cisplatin cytotoxicity. Moreover, the bystander effect of PQ1 on cisplatin reveals that PQ1 has a direct involvement in cisplatin toxicity in high cell density cultures (Figure 3C).

In addition to GJIC-dependent mechanism, PQ1 also adds tumor-suppressing component to the combinational treatment by GJIC-independent mechanisms. PQ1 inhibited cell proliferation, decreased cell viability and induced apoptosis (Figures 2,3 \& 6). The findings also showed that the cytotoxicity of PQ1 is cell density independent; supporting that PQ1 can mediate cytotoxic effect via GJIC-independent mechanism. In primary breast tumors, Cx26 has been reported to affect cellular process by GJIC-independent functions [41]. In literature, genistein and quercetin increase $\mathrm{Cx} 43$ and suppress breast cancer cell proliferation in a GJIC-independent way [42]. All these studies demonstrate that connexins can function as tumor suppressors via GJIC-independent mechanism. Therefore, the GJICindependent cytotoxicity of PQ1 may be related to the overexpression of connexins by PQ1. In the combinational treatment, $\mathrm{Cx} 26, \mathrm{Cx} 32$, and $\mathrm{Cx} 43$ protein levels were increased in the presence of PQ1 (Figure
5). $500 \mathrm{nM}$ of PQ1 not only rescued cisplatin-induced decreases of Cx26 and Cx32, but also increased Cx26 and Cx32 to a level higher than control (no treatment) (Figure 5). Cell viability results showed that PQ1 increased toxicity of cisplatin at low cell density when there is no gap junction formation (Figure 3B). Current findings established a GJIC-independent mechanism for the effect of PQ1 on cisplatinmediated response.

This report showed that combinational treatment of PQ1 and cisplatin had a synergistic effect on apoptosis by activating both the intrinsic and extrinsic apoptotic pathways (Figures 6A \& 6B). Both the bystander effect and GJIC-independent mechanism may be responsible for the apoptosis induction. The cisplatin-DNA adducts have been reported to activate caspases and induce apoptosis [5]. Therefore, one hypothesis related to bystander effect is that PQ1 restores gap junction channels in the combinational treatment, allowing increasing amounts of cisplatin-induced cytotoxic signals to enter the neighboring cells, which accordingly trigger the apoptosis by a GJIC-dependent mechanism. The other hypothesis related to GJIC-independent mechanism depends on connexins. Expressions of Cx26 and Cx43 have been reported to be correlated with the expression level of the proapoptotic factor Bax, suggesting that connexins may participate in apoptotic pathways [43]. Therefore, the second hypothesis is that apoptosis induced by combinational treatment may attribute to the overexpression of connexins in the presence of PQ1.

Cisplatin resistance has been widely reported in many cancer treatments, including testicular, ovarian, and cervical cancers. Studies of gap junctions revealed that GJIC and connexin expressions are dramatically reduced in these cancer cells. In ovarian cancer cell lines, expressions of $\mathrm{Cx} 26$ and $\mathrm{Cx} 43$ are reduced [44]. Chemosensitivity studies found that loss of $\mathrm{Cx} 43$ proteins may be associated with sensitivity to anticancer drugs [44]. In dysplastic ectocerivix, a premalignant lesion which can turn cancerous, Cx26, Cx30 and $\mathrm{Cx} 43$ display a loss of expression [45]. The characteristic loss of connexins in these cancers as well as the results of this study implies that the application of the combinational treatment of PQ1 and cisplatin can be expanded to the therapy of various cisplatin-resistant cancers.

The present study showed that combinational treatment of PQ1 and cisplatin activates the activity of gap junction channels, increases the expression of connexins, and potentiates the cytotoxicity of cisplatin by inducing apoptosis. PQ1 is a promising molecule for combinational therapy aimed at potentiating cisplatin efficacy, decreasing cisplatin resistance and reducing side effects.

\section{Acknowledgement}

We gratefully acknowledge the financial support from the NIH R15CA152922 and P30RR030926.

\section{References}

1. Rosenberg B, Vancamp L, Krigas T (1965) Inhibition of Cell Division in Escherichia Coli by Electrolysis Products from a Platinum Electrode. Nature 205: 698-699.

2. Rosenberg B, VanCamp L, Trosko JE, Mansour VH (1969) Platinum compounds: a new class of potent antitumour agents. Nature 222: 385-386.

3. Giaccone G (2000) Clinical perspectives on platinum resistance. Drugs 59: S9S17.

4. O'Reilly SE, Gelmon KA (1995) Biweekly paclitaxel and cisplatin: a phase I/II study in the first-line treatment of metastatic breast cancer. Semin Oncol 22 109-111.

5. Wang D, Lippard SJ (2005) Cellular processing of platinum anticancer drugs Nat Rev Drug Discov 4: 307-320. 
Citation: Ding Y, Nguyen TA (2012) Gap Junction Enhancer Potentiates Cytotoxicity of Cisplatin in Breast Cancer Cells. J Cancer Sci Ther 4: 371378. doi:10.4172/1948-5956.1000170

6. Cepeda V, Fuertes MA, Castilla J, Alonso C, Quevedo C, et al. (2007) Biochemical mechanisms of cisplatin cytotoxicity. Anticancer Agents Med Chem 7: 3-18

7. Wernyj RP, Morin PJ (2004) Molecular mechanisms of platinum resistance: still searching for the Achilles' heel. Drug Resist Updat 7: 227-232.

8. Simpson I, Rose B, Loewenstein WR (1977) Size limit of molecules permeating the junctional membrane channels. Science 195: 294-296.

9. Kumar NM, Gilula NB (1996) The gap junction communication channel. Cell 84 381-388.

10. Willecke K, Eiberger J, Degen J, Eckardt D, Romualdi A, et al. (2002) Structura and functional diversity of connexin genes in the mouse and human genome. Biol Chem 383: 725-737.

11. Mesnil M, Crespin S, Avanzo JL, Zaidan-Dagli ML (2005) Defective gap junctional intercellular communication in the carcinogenic process. Biochim Biophys Acta 1719: 125-145.

12. Pointis G, Fiorini C, Gilleron J, Carette D, Segretain D (2007) Connexins as precocious markers and molecular targets for chemical and pharmacological agents in carcinogenesis. Curr Med Chem 14: 2288-2303.

13. Naus CC, Laird DW (2010) Implications and challenges of connexin connections to cancer. Nat Rev Cancer 10: 435-441.

14. Jiang JX, Gu S (2005) Gap junction- and hemichannel-independent actions of connexins. Biochim Biophys Acta 1711: 208-214.

15. Eghbali B, Kessler JA, Reid LM, Roy C, Spray DC (1991) Involvement of gap junctions in tumorigenesis: transfection of tumor cells with connexin 32 cDNA retards growth in vivo. Proc Natl Acad Sci U S A 88: 10701-10705.

16. Kandouz M, Batist G (2010) Gap junctions and connexins as therapeutic targets in cancer. Expert Opin Ther Targets 14: 681-692.

17. van Dillen IJ, Mulder NH, Vaalburg W, de Vries EF, Hospers GA (2002) Influence of the bystander effect on HSV-tk/GCV gene therapy. A review. Curr Gene Ther 2: 307-322.

18. Prise KM, O'Sullivan JM (2009) Radiation-induced bystander signalling in cancer therapy. Nat Rev Cancer 9: 351-360.

19. Merle P, Morvan D, Caillaud D, Demidem A (2008) Chemotherapy-induced bystander effect in response to several chloroethylnitrosoureas: an origin independent of DNA damage? Anticancer Res 28: 21-27.

20. Trosko JE, Chang CC (2001) Mechanism of up-regulated gap junctional intercellular communication during chemoprevention and chemotherapy of cancer. Mutat Res 480-481: 219-229.

21. Jensen R, Glazer PM (2004) Cell-interdependent cisplatin killing by Ku/DNAdependent protein kinase signaling transduced through gap junctions. Proc Natl Acad Sci U S A 101: 6134-6139.

22. Peterson-Roth E, Brdlik CM, Glazer PM (2009) Src-Induced cisplatin resistance mediated by cell-to-cell communication. Cancer Res 69: 3619-3624.

23. He B, Tong X, Wang L, Wang Q, Ye H, et al. (2009) Tramadol and flurbiprofen depress the cytotoxicity of cisplatin via their effects on gap junctions. Clin Cancer Res 15: 5803-5810.

24. Wang Q, You T, Yuan D, Han X, Hong X, et al. (2010) Cisplatin and oxaliplatin inhibit gap junctional communication by direct action and by reduction of connexin expression, thereby counteracting cytotoxic efficacy. J Pharmaco Exp Ther 333: 903-911.

25. Gakhar G, Ohira T, Shi A, Hua DH, Nguyen TA (2008) Antitumor effect of substituted quinolines in breast cancer cells. Drug Dev Res 69: 526-534.

26. Gakhar G, Hua DH, Nguyen TA (2010) Combinational treatment of gap junctional activator and tamoxifen in breast cancer cells. Anticancer Drugs 21 : $77-88$

27. Shi A, Nguyen TA, Battina SK, Rana S, Takemoto DJ, et al. (2008) Synthesis and anti-breast cancer activities of substituted quinolines. Bioorg Med Chem Lett 18: 3364-3368.

28. Ott I, Gust R (2007) Preclinical and clinical studies on the use of platinum complexes for breast cancer treatment. Anticancer Agents Med Chem 7: 95110.

29. McLachlan E, Shao Q, Laird DW (2007) Connexins and gap junctions in mammary gland development and breast cancer progression. J Membr Bio 218: 107-121.

30. Eastman A, Lippert B (1999) The mechanism of action of cisplatin: From adducts to apoptosis, in Cisplatin. Chemistry and Biochemistry of a Leading Anticancer Drug: 111-134.

31. Seki K, Yoshikawa H, Shiiki K, Hamada Y, Akamatsu N, et al. (2000) Cisplatin (CDDP) specifically induces apoptosis via sequential activation of caspase-8, -3 and -6 in osteosarcoma. Cancer Chemother Pharmacol 45: 199-206.

32. Kojima H, Endo K, Moriyama H, Tanaka Y, Alnemri ES, et al. (1998) Abrogation of mitochondrial cytochrome $c$ release and caspase- 3 activation in acquired multidrug resistance. J Biol Chem 273: 16647-16650.

33. Mueller T, Voigt W, Simon H, Fruehauf A, Bulankin A, et al. (2003) Failure of activation of caspase-9 induces a higher threshold for apoptosis and cisplatin resistance in testicular cancer. Cancer Res 63: 513-521.

34. Fisher DE (1994) Apoptosis in cancer therapy: crossing the threshold. Cell 78 539-542.

35. Jones NA, Turner J, Mcllwrath AJ, Brown R, Dive C (1998) Cisplatin- and paclitaxel-induced apoptosis of ovarian carcinoma cells and the relationship between bax and bak up-regulation and the functional status of p53. Mo Pharmacol 53: 819-826.

36. Henkels KM, Turchi JJ (1999) Cisplatin-induced apoptosis proceeds by caspase-3-dependent and -independent pathways in cisplatin-resistant and -sensitive human ovarian cancer cell lines. Cancer Res 59: 3077-3083.

37. Eliopoulos AG, Kerr DJ, Herod J, Hodgkins L, Krajewski S, et al. (1995) The control of apoptosis and drug resistance in ovarian cancer: influence of p53 and Bcl-2. Oncogene 11: 1217-1228.

38. Kartalou M, Essigmann JM (2001) Mechanisms of resistance to cisplatin. Mutat Res 478: 23-43.

39. Ding Y, Prasain K, Nguyen TD, Hua DH, Nguyen TA (2012) The effect of the PQ1 anti-breast cancer agent on normal tissues. Anticancer Drugs 23: 897 905

40. Leithe E, Rivedal E (2007) Ubiquitination of gap junction proteins. J Membr Bio 217: 43-51.

41. Kalra J, Shao Q, Qin H, Thomas T, Alaoui-Jamali MA, et al. (2006) Cx26 inhibits breast MDA-MB-435 cell tumorigenic properties by a gap junctional intercellula communication-independent mechanism. Carcinogenesis 27: 2528-2537.

42. Conklin CM, Bechberger JF, MacFabe D, Guthrie N, Kurowska EM, et al (2007) Genistein and quercetin increase connexin43 and suppress growth of breast cancer cells. Carcinogenesis 28: 93-100.

43. Kanczuga-Koda L, Sulkowski S, Tomaszewski J, Koda M, Sulkowska M, et al. (2005) Connexins 26 and 43 correlate with Bak, but not with Bcl-2 protein in breast cancer. Oncol Rep 14: 325-329.

44. Toler CR, Taylor DD, Gercel-Taylor C (2006) Loss of communication in ovarian cancer. Am J Obstet Gynecol 194: e27-31.

45. Aasen T, Graham SV, Edward M, Hodgins MB (2005) Reduced expression of multiple gap junction proteins is a feature of cervical dysplasia. Mol Cancer 4 31. 